\title{
IMPROVING THE HEALTH BEHAVIOURS OF COPD PATIENTS: IS HEALTH LITERACY THE ANSWER?
}

\author{
Tracy A. Freeze ${ }^{1}$, Leanne Skerry ${ }^{2}$, Emily Kervin ${ }^{3}$, Andrew Brillant ${ }^{4}$, \\ Jennifer Woodland ${ }^{1}$, \& Natasha Hanson ${ }^{1}$ \\ ${ }^{1}$ Ph.D., Research Services, Horizon Health Network (Canada) \\ ${ }^{2}$ M.A., Research Services, Horizon Health Network (Canada) \\ ${ }^{3}$ M.A.(Cand.), Research Services, Horizon Health Network (Canada) \\ ${ }^{4}$ B.Pharm., Pharmacy, Horizon Health Network (Canada)
}

\begin{abstract}
Chronic Obstructive Pulmonary Disease (COPD) is a leading cause of morbidity and mortality and contributes to substantial social and economic burden (Vogelmeier et al., 2017). There is no cure for COPD, however, medications are available which slow disease progression and control symptoms. Adherence to prescribed medications is critical for optimal management of the disease as is the proper use of the medication delivery device. O'Conor et al. (2019) found that lower health literacy was associated with both poor medication adherence and poor inhalation technique. Health literacy, according to the Process-Knowledge Model, consists of both processing capacity and knowledge (Chin et al., 2015). COPD most commonly occurs in older adults (Cazzola, Donner, \& Hanania, 2007). Older adults tend to have lower processing capacity, but lower processing capacity can be mitigated by knowledge (Chin et al., 2015). The purpose of this study was to determine if health literacy was associated with medication adherence and/or inhalation technique. Fifty-seven participants (age range 55-94 years) completed a questionnaire package that included the REALM, TOFHLA, and demographic questions. Information was gathered on medication refill adherence and inhalation technique. A subset of twenty COPD patients participated in qualitative interviews. Results indicated that lower health literacy was associated with both lower medication adherence and poor inhalation technique. One of the themes expressed by the qualitative participants was the need for further information. Given that health literacy is associated with health behaviours in older adults with COPD and there is an expressed need for information, an example of how current educational materials may be reformatted to meet the lower processing capacity of older adults will be discussed. An action-oriented research project where pharmacists and COPD patients collaborate to design needed educational materials and interventions is suggested as a next step.
\end{abstract}

Keywords: Health literacy, health behaviours, cognition, older adults.

\section{Introduction}

Chronic Obstructive Pulmonary Disease (COPD) is a globally prevalent chronic disease that results in an obstruction in airflow from the lungs and varying respiratory symptoms (Vogelmeier, 2017). COPD is a leading cause of morbidity and mortality globally and contributes to substantial social and economic burden (WHO, 2010). Both medication adherence and adequate inhalation technique are essential for controlling symptoms and slowing COPD disease progression (Rodriguez-Rodin, 2005; Melani et al, 2011). Nevertheless, rates of medication adherence and adequate inhalation technique remain low (see Restrepo et al., 2008). The purpose of the current research was to determine if health literacy was associated with medication adherence and/or inhalation technique in a sample of older adults and to propose next steps for targeted educational materials and interventions.

Health literacy can be defined as "the ability to access, understand, evaluate and communicate information as a way to promote, maintain and improve health in a variety of settings across the life-course" (Rootman \& Gordon-El-Bihbety, 2008, p.11). It is estimated that 55\% of Canadians aged 16 to 65 have low health literacy, with approximately $88 \%$ of individuals over the age of 65 having low health literacy (Rootman \& Gordon-El-Bihbety, 2008). Concerning COPD specifically, Omachi et al. (2012) found that patients with low levels of health literacy may be at increased risk for poor health outcomes. Further, health literacy, according to the Process-Knowledge Model, consists of both processing capacity and knowledge (Chin et al., 2015). Knowledge about a disease and the respective treatment is essential, but the effective delivery of knowledge may be even more important if a patient has lower processing capacity. 
Processing capacity involves cognitive processes such as working memory and processing speed. According to Chin et al. (2015), knowledge and processing capacity have two different age-related trajectories with processing capacity declining with age and knowledge remaining constant or increasing with age. Recently, O'Conor et al., (2019) conducted an observational cohort study to determine if health literacy and cognitive abilities were associated with the health behaviours of COPD patients. They found that health literacy and cognitive abilities were associated with both medication adherence and inhalation technique. Further, they found that fluid abilities (e.g., processing capacity), but not crystallized abilities (e.g., knowledge), were associated with health behaviours.

Given that COPD most commonly occurs in older adults (Cazzola, Donner, \& Hanania, 2007), health literacy is lowest in older adults (Rootman \& Gordon-El-Bihbety, 2008), and processing capacity decreases in older adults, the current research hypothesized that lower levels of health literacy would be associated with lower levels of medication adherence and inhalation technique in older adults.

\section{Methods}

\subsection{Participants}

See Table 1 for a description of all study participants compared to the qualitative interview subsample. Participants' ages ranged from 55 to 94 years.

Table 1. Descriptive Statistics for All Participants Compared to Interview Subsample.

\begin{tabular}{|c|c|c|}
\hline Variable & All $(n=57)$ & Interview $(n=20)$ \\
\hline Age, M(SD) & $70.84(9.51)$ & $67.7(7.02)$ \\
\hline \multicolumn{3}{|l|}{ Gender, \% } \\
\hline Male & 36.8 & 35.0 \\
\hline Female & 63.2 & 65.0 \\
\hline \multicolumn{3}{|l|}{ Education, \% } \\
\hline$<$ Grade 8 & 24.5 & 5.0 \\
\hline$<$ High school & 21.1 & 20.0 \\
\hline High school & 42.1 & 60.0 \\
\hline College/University & 12.3 & 15.0 \\
\hline \multicolumn{3}{|l|}{ Current Smoker, \% } \\
\hline Yes & 33.3 & 25.0 \\
\hline \multicolumn{3}{|l|}{ TOFHLA ${ }^{\mathrm{a}}, \%$} \\
\hline Inadequate & 38.6 & 35.0 \\
\hline Adequate & 56.1 & 65.0 \\
\hline \multicolumn{3}{|l|}{ REALM $^{\mathrm{b}}, \%$} \\
\hline Inadequate & 38.6 & 25.0 \\
\hline Adequate & 59.6 & 75.0 \\
\hline \multicolumn{3}{|l|}{$\mathbf{M R A}^{\mathbf{c}}, \%$} \\
\hline Sub-Optimal & 40.4 & 40.0 \\
\hline Optimal & 56.1 & 60.0 \\
\hline \multicolumn{3}{|l|}{ MIT $^{\mathrm{d}}, \%$} \\
\hline Poor Technique & 10.5 & 5.0 \\
\hline Good Technique & 82.5 & 95.0 \\
\hline
\end{tabular}

a TOFHLA $=$ Test of Health Literacy in Adults; ${ }^{\mathrm{b}}$ REALM $=$ Rapid Estimate of Adult Literacy in Medicine; ${ }^{\mathrm{c}}$ MRA = Medication Refill Adherence; ${ }^{\mathrm{d}}$ MIT = Inhalation Technique. Note: The discrepancies in totals reflect missing values

\subsection{Measures}

Participant demographics. Participants completed a nine-item questionnaire that included questions regarding age, gender, income, marital status, living arrangement, employment, education, years using a primary inhaler, and smoking history.

Rapid Assessment of Literacy in Medicine (REALM; Davis et al., 1993). The REALM was used to determine the participants' ability to read health terms. The REALM is a 66-item health-related word recognition test where the words are arranged in order of easy (i.e., fat) to increasing difficulty (i.e., Osteoporosis). For this research, the REALM was collapsed into two categories: inadequate ( $\leq 6^{\text {th }}$ grade) and adequate $\left(\geq 9^{\text {th }}\right.$ grade). The REALM had good internal reliability $(\alpha=.98)$.

Test of Functional Health Literacy in Adults (TOFHLA; Baker et al, 1999). The TOFHLA measured the participants' ability to understand health information. The TOFHLA consisted of a two-part assessment; part one provided the participant with medical information or instructions from which they answered questions to test their understanding of the information, and part two required participants to read text about medical topics and fill in the blanks of the text from a list of potential choices. For this research, TOFHLA had two categories: inadequate (0-74) and adequate (75-100). The TOFHLA had good internal reliability $(\alpha=.81)$. 
COPD Medication Refill Adherence (MRA). Information regarding the participant's primary inhaler was gathered from pharmacy records. Participants were classified (see Hess, Raebel, Conner, and Malone, 2006) as having either optimal MRA $(\geq 80)$ or sub-optimal MRA $(<80)$. For participants who were prescribed more than one medication for COPD, the MRA score was calculated for their primary inhaler.

COPD Medication Inhalation Technique (MIT). Inhalation checklists were used to assess MIT for individuals who were using either a metered-dose inhaler (MDI) or the HandiHaler (HH). As different steps are required for each device, different checklists were used to score inhalation technique depending on the type of device they were using. Each checklist contained five critical errors. Participants were determined to have poor technique if they did not perform all of the essential steps correctly and good technique if they performed all of the essential steps correctly. These checklists were based on arbitrary criteria related to the proper technique of using an inhalation device and were developed by Melani et al. (2011). The initial Intraclass Correlation Coefficient of the first 30 inhalation technique videos did not meet the minimum criteria of .7. Raters met to discuss areas of disagreement and completed the remaining videos. The final inter-rater reliability was 1.0.

\subsection{Procedure}

Ethics approval was obtained by the Horizon Research Ethics Board before recruitment. Participants were recruited by 1) using pharmacy patient records from New Brunswick pharmacy databases; and, 2) recruiting participants themselves directly to the study by advertising the study online, in newspapers, on the radio, and other public places (e.g., COPD support groups). Patients were invited to participate in the study by the pharmacist and/or pharmacist-assistant if they were at least 40 years of age, had a smoking history, a self-report diagnosis of COPD, and a targeted inhaler scheduled for daily use for at least one year. Only participants who had been prescribed a MDI or a HH were included in the study. Participants were excluded from participation if they had an asthma diagnosis, had been enrolled in a formal disease self-management program, if they had refilled their medication(s) at more than one pharmacy within the past year, or if they were prescribed three or more of the targeted inhalers for scheduled daily use. Once permission to contact and eligibility were established, a research assistant collected the measures and inhalation technique video from participants at their homes.

Finally, qualitative semi-structured interviews were conducted with a self-identified subsample of 20 participants. All those participants that consented (at the time of the initial questionnaire) to being contacted for an interview were then telephoned by research team members for further oral consent, upon review of the consent form (emailed or mailed to participants). The interviews were conducted, by research team members, over the telephone and were digitally recorded. The interview questions pertained to their experiences having COPD, adhering to medication, and what could facilitate adherence. The interview guide was piloted with two participants and, the questions were adjusted to gain as much information as possible, with possible lower literacy levels taken into account. The interviews were transcribed verbatim and uploaded to NVivo. Iterative thematic analysis (Braun \& Clarke, 2006) was conducted on the qualitative data by three members of the research team (NH, LS, EK). Consensus of themes was reached between team members.

\section{Results}

\subsection{Quantitative}

Two $2 \times 2$ chi-squares were used to analyze the relationship between health literacy and MRA. There was a significant association between health literacy, as measured by TOFHLA and REALM, and MRA (see Figure 1 and Table 2). The odds of adequate REALM was $4.67(95 \% \mathrm{CI}=1.47,14.86)$ times higher for the group with optimal MRA. Similarly, the odds of adequate TOFHLA was $7.35(95 \%$ $\mathrm{CI}=2.15,25.14$ ) times higher in the optimal MRA group.

Figure 1. Bar Graph of REALM and TOFHLA by MRA.

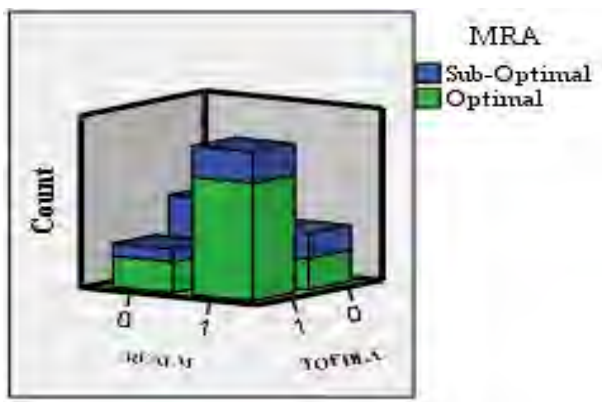


An additional two 2 × 2 chi-squares were conducted to analyze the relationship between health literacy and MIT. There was a significant association between health literacy, as measured by TOFHLA and REALM, and MIT (see Table 2). The odds ratios for MIT and health literacy could not be calculated given that none of the COPD participants with adequate health literacy had poor MIT. In both chi-squares, $50 \%$ of the cells had expected values $<5$. Even though Fisher's exact test was used, the result for MIT must be interpreted with caution. It should also be noted that, because four tests were conducted, a Bonferroni correction was applied and the significance level was set to .0125 .

Table 2. Results of Chi-Square Test for Healthy Literacy, Medication Adherence, and Inhalation Technique.

\begin{tabular}{|c|c|c|c|c|c|c|c|c|}
\hline & \multicolumn{4}{|c|}{ TOFHLA $^{\mathrm{a}}$} & \multicolumn{3}{|c|}{ REALM $^{\text {b }}$} & \multirow{3}{*}{ CI } \\
\hline & $X^{2}$ & Cramer's V & OR & CI & $X^{2}$ & Cramer's V & $\overline{\text { OR }}$ & \\
\hline $\mathbf{M R A}^{\mathbf{c}}$ & $11.02^{* * *}$ & .46 & & & $7.17^{* *}$ & .36 & & \\
\hline Sub-Optimal & & & 3.02 & $1.48-6.15$ & & & 2.33 & $1.23-4.43$ \\
\hline Optimal & & & .41 & $0.22-0.78$ & & & .5 & $0.28-0.90$ \\
\hline $\mathbf{M I T}^{\mathbf{d}}$ & $7.07^{\mathrm{e}}$ & .38 & & & $9.25^{f}$ & .42 & & \\
\hline Poor & & & & & & & & \\
\hline Good & & & .77 & $0.62-0.97$ & & & .73 & $0.56-0.94$ \\
\hline
\end{tabular}

\subsection{Qualitative}

One of the themes that emerged from the thematic analysis of the qualitative data was directly related to health literacy and the formulation of educational materials ${ }^{1}$. The theme is "there needs to be more information," in which many participants (12/20) expressed that they desired more information, including at the time of diagnosis, about the disease itself, how they can impact disease progression, how to get help when needed, and the supports available. Regarding what they perceived to be the overall need for more information one participant stated, "my big concern is on the COPD end of it is the lack of information that's available to anybody that has the particular problem"(P29). When asked what information they were provided with upon diagnosis one participant stated, "Um, really nothin'. They just come in and told me I had COPD and gave these puffers and, that was it." (P42). There were also participants who specifically wanted to know what to expect as their disease progressed, "You know, what... what signs there is, like, when um, when I'm talking a lot you might notice my voice gets different and I think that must be from the COPD?...I don't know if it is, but if it is, people, they should tell you what, what to expect."(P4).

\section{Discussion}

Supporting the findings of O'Conor et al. (2019), we found that a lower level of health literacy was associated with lower levels of both MRA and MIT. COPD patients with low MRA and MIT are at risk for uncontrolled COPD symptoms and advancing disease progression (Rodriguez-Rodin, 2005; Melani et al, 2011). Further, one of the themes that emerged from the qualitative interviews was the need for more information about the prognosis, management, and treatment of COPD. Providing COPD information repeatedly and over the progression of the disease would address the informational needs of COPD patients as identified by our sample. Though increasing knowledge will help improve health literacy, it is only one part of health literacy. Processing capacity also needs to be considered. Indeed, it may be crucial when the primary age group of the disease is older adults. Older adults are most likely to be affected by a decline in cognitive abilities such as processing speed (e.g., O'Conor et al., 2019). Therefore, the information that is provided to older adults needs to be tailored to their needs. In other words, information on COPD needs to improve not only in quantity but also in quality.

O'Conor et al. (2019) offered some suggestions. They noted that COPD patient materials may be improved by designing informational materials for the low reading grade level and providing concrete instructions (p. 24). They also noted that multi-step behaviours, like MIT, should be 'chunked' to enhance memory (e.g., "Ready, Set, Go!", p. 24). Chin et al. (2017) noted that materials should signal key concepts and present the information in a way that reduces the demands on processing skills like search and reorganization. Also, designers of COPD patient materials may consider the importance of information delivered via the internet. Morrow and colleagues (e.g., 2016, 2017) offered many important insights into how to deliver effective electronic health information to older adults with low health literacy.

\footnotetext{
${ }^{1}$ The full qualitative study will be published at a later date. In addition to the major theme described in this article, the other major themes in the qualitative study included: "continuity of care", "drug costs a concern", "importance of pharmacy for information and care", "medication self-management", and "it's a lonely battle”.
} 
Finally, the most efficacious method may be an action-oriented research project where pharmacists, cognitive psychologists, and COPD patients collaborate to design needed educational materials and interventions.

Though this research is limited by the sample size and initial issues with MIT coding, other researchers (e.g., O'Conor et al., 2019) have reported similar results. Future research should focus on designing and/or re-formatting and testing COPD patient materials with a focus on the specific needs of older adult COPD patients.

\section{Acknowledgements}

This research was funded by AstraZeneca, the New Brunswick Health Research Foundation, and the New Brunswick Pharmacists' Association.

\section{References}

Baker, D. W., Williams, M. V., Parker, R. M., Gazmararian, J. A., \& Nurss, J. (1999). Development of a brief test to measure functional health literacy. Patient Education and Counseling, 38(1), 33-42.

Braun, V., \& Clarke, V. (2006). Using thematic analysis in psychology. Qualitative research in psychology, 3(2), 77-101.

Cazzola, M., Donner, C. F., \& Hanania, N. A. (2007). One hundred years of chronic obstructive pulmonary disease (COPD). Respiratory Medicine, 101, 1049-1065.

Chin, J., Madison, A., Gao, X., Graumlich, J. F., Conner-Garcia, T., Murray, M. D., ... \& Morrow, D. G. (2017). Cognition and health literacy in older adults' recall of self-care information. The Gerontologist, 57(2), 261-268.

Davis, T. C., Long, S. W., Jackson, R. H., Mayeaux, E. J., George, R. B., Murphy, P. W., \& Crouch, M. A. (1993). Rapid estimate of adult literacy in medicine: a shortened screening instrument. Family Medicine, 25(6), 391-395.

Hess, L. M., Raebel, M. A., Conner, D. A., \& Malone, D. C. (2006). Measurement of adherence in pharmacy administrative databases: a proposal for standard definitions and preferred measures. Annals of pharmacotherapy, 40(7-8), 1280-1288.

Melani, A.S., Bonavia, M., Cilenti, V., Cinti, C., Lodi, M., Martucci, P., ... \& Neri, M. (2011). Inhaler mishandling remains common in real life and is associated with reduced disease control. Respiratory Medicine, 105(6), 930-938. doi: 10.1016/j.rmed.2011.01.005

Morrow, D. (2016). Technology-based support for older adult communication in safety-critical domains. In Psychology of Learning and Motivation (Vol. 64, pp. 285-317). Academic Press.

Morrow, D., Hasegawa-Johnson, M., Huang, T., Schuh, W., Azevedo, R. F. L., Gu, K., ... \& Garcia-Retamero, R. (2017). A multidisciplinary approach to designing and evaluating Electronic Medical Record portal messages that support patient self-care. Journal of Biomedical Informatics, 69, 63-74.

O'Conor, R., Muellers, K., Arvanitis, M., Vicencio, D. P., Wolf, M. S., Wisnivesky, J. P., \& Federman, A. D. (2019). Effects of health literacy and cognitive abilities on COPD self-management behaviors: A prospective cohort study. Respiratory Medicine, 160, 105630.

Omachi, T. A., Sarkar, U., Yelin, E. H., Blanc, P. D., \& Katz, P. P. (2013). Lower health literacy is associated with poorer health status and outcomes in chronic obstructive pulmonary disease. Journal of General Internal Medicine, 28(1), 74-81

Restrepo, R. D., Alvarez, M. T., Wittnebel, L. D., Sorenson, H., Wettstein, R., Vines, D. L., ... \& Wilkins, R. L. (2008). Medication adherence issues in patients treated for COPD. International Journal of Chronic Obstructive Pulmonary Disease, 3(3), 371.

Rodríguez-Roisin, R. (2005). The airway pathophysiology of COPD: implications for treatment. COPD: Journal of Chronic Obstructive Pulmonary Disease, 2(2), 253-262.

Rootman, I., \& Gordon-El-Bihbety, D. (2008). A vision for a health literate Canada. Ottawa, ON: Canadian Public Health Association.

Vogelmeier, C. F., Criner, G. J., Martinez, F. J., Anzueto, A., Barnes, P. J., Bourbeau, J., ... \& Frith, P. (2017). Global strategy for the diagnosis, management, and prevention of chronic obstructive lung disease 2017 report. GOLD executive summary. American Journal of Respiratory and Critical Care Medicine, 195(5), 557-582.

World Health Organization. Global status report on noncommunicable diseases 2010. Geneva, 2011. Available at http://www.who.int/nmh/publications/ncd_report2010/en/ Accessed January 21, 2020. 